\title{
ANALYSIS AND OPTIMISATION OF LOOPED WATER DISTRIBUTION NETWORKS
}

\author{
BRIAN YOUNG ${ }^{1}$
}

(Received 12 March 1997; revised 23 February 1998)

\begin{abstract}
A three stage procedure for the analysis and least-cost design of looped water distribution networks is considered in this paper. The first stage detects spanning trees and identifies the true global optimum for the system. The second stage determines hydraulically feasible pipe flows for the network by the numerical solution of a set of non-linear simultaneous equations and shows that these solutions are contained within closed convex polygonal regions in the solution space bounded by singularities resulting from zero flows in individual pipes. Ideal pipe diameters, consistent with the pipe flows and the constant velocity constraint adopted to prevent the system degenerating into a branched network, are selected and costed. It is found that the most favourable optimum is in the vicinity of a vertex in the solution space corresponding to the minimum spanning tree. In the third stage, commercial pipes are specified and the design finalised. Upper bound formulae for the number of spanning trees and hydraulically feasible solutions in a network have also been proposed. The treatment of large networks by a heuristic procedure is described which is shown to result in significant economies compared with designs obtained by non-linear programming.
\end{abstract}

\section{Introduction}

Water distribution networks consist of a planar system of pipes or links (through which the water flows), connected together at nodes which may be at different elevations. In general, the complex will also include pumps, reservoirs and valves. A node usually has one of two main functions; it either receives a supply for the system or it delivers the demand required by consumers. As a special case, it may satisfy neither of these requirements but merely serve as a junction between two or more pipes. The pressure head at a supply node is established by the presence of a pump or a reservoir. Resistances to flow (friction losses) which are a function of length, diameter, flow

\footnotetext{
${ }^{1}$ Department of Civil Engineering, Papua New Guinea University of Technology, Lae, Papua New Guinea.

(C) Australian Mathematical Society 2000, Serial-fee code 0334-2700/00
} 
rate, and pipe material and roughness, occur in the links as the fluid flows around the network from supply nodes to demand nodes. The effect of minor losses may be included as equivalent pipe lengths. It is usual to specify a minimum acceptable residual pressure head at demand nodes and the pressure heads at supply nodes must be of sufficient magnitude to satisfy these requirements. The difference between the total heads (measured with reference to a common horizontal datum) at a supply node and a demand node is equal to the algebraic sum of the head losses taken along any path in the network.

Networks are of two different types; branched or open tree-like networks and closed-looped networks. Branched networks have no built-in redundancy and, if one of the links fails, no service is available at downstream nodes. For this reason looped networks, which permit demand nodes to be served through more than one path, are preferred for urban water supply.

There is only one flow solution for a branched network, but many solutions are possible for looped systems, all of which satisfy the general closed network laws of continuity and energy balance. The number of such solutions depends on the number of links in each loop and the configuration of the network. The analysis of looped networks with known pipe diameters, although a non-linear problem due to the nature of the pipe resistance law, is not hard provided the network is relatively small. For bigger networks, technical difficulties arise because of the need to deal with large sparse matrices. The general design problem however, which requires the selection of pipe sizes, is much more arduous since it includes both analysis and optimisation subject to certain criteria such as least-cost.

Design may involve a completely new network, or the expansion and/or upgrading of an existing network. Decision variables may be chosen as pipe diameters, pipe flows, or pressures at demand nodes. Solutions to the general design problem are local optima and the ideal outcome is to find the most favourable local optimum, usually known as the global optimum. Paradoxically, the true global optimum for a looped network is a particular branched network (a minimum spanning tree or the least-cost branched network which serves all the nodes of the original system). It is therefore necessary to force the network to retain its looped structure by the introduction of artificial constraints on the solution such as specified minimum pipe diameters or flows or, as in the present paper, by control of the flow velocity. Since the true global optimum solution is no longer applicable, the best result that can be expected is the identification of a favourable local optimum consistent with satisfaction of the additional constraints.

Optimisation by non-linear programming is generally defined as the attainment of an extremum for the objective function (in this case, a minimum of the cost function). It will be shown here that optimal cost does not correspond to a mathematical minimum but is simply the least of the costs associated with each of the hydraulically feasible 
solutions. Furthermore, the solution space contains as many singularities as there are links in the network; thus the cost function cannot be treated as continuously differentiable.

The approach to determining economic solutions for looped water distribution networks, presented in this paper, involves three stages. The first stage leads to identification of the minimum spanning tree and only requires knowledge of the network geometry and satisfaction of the continuity equations for the network as a whole and at each node. The second stage determines hydraulically feasible flows for the intact network which are likely to be favourable optima, by numerical solution of the loop head-loss equations using starting values in the vicinity of the minimum spanning tree. In order to simplify the determination of ideal (continuous) least-cost pipe diameters and to force the solution away from degeneration into a branched network, the flow velocity is initially assumed to be constant for all links. This has the important advantage that the head loss in a link may be expressed in terms of a single variable (the pipe flow). As a further preliminary simplification, all links are presumed to be of the same material and roughness. The third stage of the solution completes the design; stock pipe sizes are selected using corresponding ideal diameters as a guide, new balancing flows are calculated, different types of pipe are allowed for as necessary and actual flow velocities in each link are obtained such that minimum node-residual-head requirements are satisfied.

The constant-velocity constraint is a realistic alternative to the imposition of minimum diameters or flows and has the additional virtue that the magnitude of the velocity may be selected to control minimum service heads at the nodes and the total cost of the pipes without affecting the flows. The constraint does not impose a serious restriction on the validity of the solution since the flow velocity would be required anyway to lie within a fairly narrow range ( $>0.5 \mathrm{~m} / \mathrm{s}$ to avoid silting and $<2.5 \mathrm{~m} / \mathrm{s}$ to avoid scouring). Indeed, Webber [14] has recommended an even narrower range (from 0.6 to $1.2 \mathrm{~m} / \mathrm{s}$ ) on both operational and economic grounds.

\section{Previous work}

There is an extensive literature on the analysis and optimisation of looped water distribution networks dating from the late 1960s. Direct and indirect methods incorporating both non-linear programming and less conventional methods have been proposed. Only seminal research which has a bearing on the present study is discussed here.

In an early work, Jacoby [6] employed an unconstrained non-linear programming approach with a gradient search technique to minimise the objective function. The search methods included negative gradient direction, random direction and a combi- 
nation of both. The pipe cost was assumed to be linear with respect to the diameters. Kesavan and Chandrashekar [7] used linear graph theory to find pipe flows in existing networks for which pipe sizes were known. Their results were compared with those obtained using the Hardy-Cross method.

Watanatada [13] presented a conventional non-linear optimisation procedure in which inequality constraints were converted to equality constraints by standard methods. The Lagrangian was minimised using gradient balancing with an exterior point penalty function. It was assumed that there were no singularities in the solution space and that only one local minimum exists. It was found that the solution degenerated into a branched network unless additional constraints defining minimum pipe diameters were adopted. The method was illustrated with reference to a simple two-loop triangular network. In a later section of the present paper, Watanatada's results for this network will be compared with solutions obtained from the alternative approach described herein.

Shamir [11] used a non-linear programming scheme which was an extension of a network solver routine employing the generalised Newton-Raphson method. Ordered triangular factorisation was employed to deal efficiently with inversion of the sparse Jacobian found for networks of practical size. The initial capital cost of the system was included in the objective function together with operating costs under one or several loadings. The solution was based on the generalised reduced gradient method and the objective function was expressed in terms of Lagrangian multipliers. Constraints on dependent variables were dealt with either by internal penalty functions, which require intervention by the user, or by variable exchange. Epp and Fowler [3] discussed the analysis of existing networks and proposed banding of the Jacobian by a judicious numbering of loops.

Rasmusen [10] also separated the network optimisation problem into analysis and design. Instead of conventional optimisation, a heuristic method was used for selecting pipe diameters in discrete sizes. Chiplunkar et al. [2] have treated the analysis and design problem separately. The analysis stage used linear graph theory in combination with Newton's method to provide fast convergence. An unconstrained non-linear optimisation program with interior penalty functions was then solved by the DavidonFletcher-Powell method. The objective function to be minimised included the costs of the pipes, pumping plant, power consumption, labour and reservoirs. Gupta et al. [5] have applied similar techniques to the expansion and upgrading of a system with multiple sources. Initial values for the solution were obtained by consideration of the minimum spanning tree for the network (although the method used to identify the minimum from the approximately $6 \times 10^{6}$ spanning trees in their example is not described). A minimum value for the pipe diameters was specified in order to ensure that the network did not degenerate into a branched system.

Quindry et al. [9] postulated linear optimisation using gradient search based on the 
assumption that the ratio of the quantity flowing in a pipe to its cost is independent of the diameter. Morgan and Goulter [8] made use of the same linear assumption to determine least-cost layouts for looped networks. Templeman [12] was critical of the linear approach and demonstrated that the looped network optimisation problem is NP-hard, and that a rigorous algorithm to find an optimum design using discrete diameters is not a practical possibility. The optimisation process tends to remove the redundancy which is the essential feature of a looped system thus the inevitable result of optimising a looped network without the inclusion of artificial constraints is a branched system.

Bhave [1] also observed that, unless minimum diameter or minimum discharge constraints are applied, the optimisation algorithm causes a looped network to degenerate into a branched system. The optimal expansion of existing water distribution systems subjected to single loading was considered by first identifying a supposedly least-cost branching configuration using a 'shortest path' algorithm. A solution was obtained, not by non-linear programming, but by an iterative procedure in which node pressure heads were taken as decision variables. Featherstone and El-Jumaily [4] took account of initial capital costs and fixed and variable annual operating costs. They also used an iterative optimisation technique, but based on the concept of dummy hydraulic gradients.

\section{An illustrative example}

Before embarking on a detailed exposition of the present theory, it will be instructive to illustrate the three stages of the optimisation process by means of a simple example. The example chosen is the analysis and least-cost design of the ring main shown in Figure 1. Pipe lengths, supply and demand flows, and node elevations are shown on the diagram; the algebraic sum of the supply and demand flows is zero. A supply pressure head of $8 \mathrm{~m}$ at node 1 is provided by a reservoir. We seek the most economic choice of commercial pipe sizes such that a minimum residual head criterion of $1.2 \mathrm{~m}$ is satisfied at all nodes. The capital costs of a pump and of building the elevated reservoir, together with the recurrent costs of pumping, maintenance and so on, are disregarded here in the interests of simplicity.

3.1. First stage. The system consists of eight links and eight nodes forming one loop. The directions of the unknown link flows are assumed to be positive if acting clockwise around the loop. Since there is one loop there is one independent link flow which may be chosen arbitrarily. If the flow $(x \mathrm{l} / \mathrm{s})$ in link 1 is selected, conservation of continuity at the nodes allows each of the other link flows to be expressed in terms of $x$ :

$$
q_{2}=x-12, \quad q_{3}=x-34, \quad q_{4}=x-50, \quad q_{5}=x-69,
$$




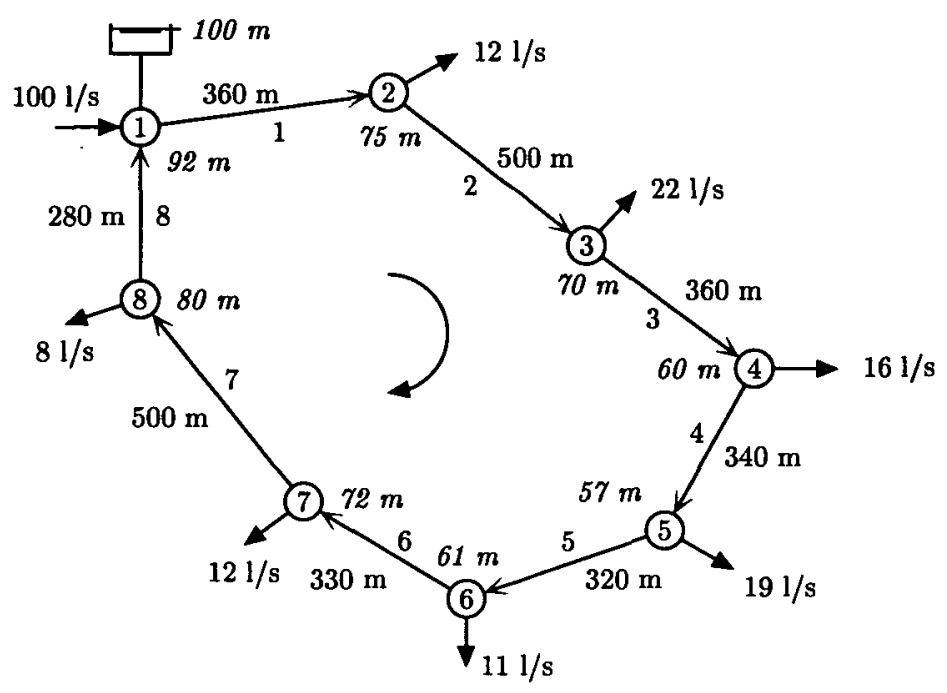

FIGURE 1. Layout of ring main.

$$
q_{6}=x-80, \quad q_{7}=x-92 \text { and } q_{8}=x-1001 / \mathrm{s} .
$$

Another consequence of the network having a single loop is that a branched system results if any one of the eight link flows is zero (if two or more non-adjacent link flows are zero simultaneously, the network becomes disjoint). Thus the singularities (giving rise to the eight viable spanning trees) in the one-dimensional solution space defined by $x$ are given by $x=0,12,34,50,69,80,92$ and $100 \mathrm{l} / \mathrm{s}$.

The true global optimum (least-cost) configuration for the ring main may be determined if data is available relating pipe cost to the corresponding flow. This type of information is obtained from manufacturer's catalogues, but in the form of cost per unit length for specific nominal diameters. A cost function of the following form is typical:

$$
C_{j}=K L_{j} d_{j}^{\beta},
$$

where $C_{j}$ is the capital cost of link $j, L_{j}$ is the length of link $j(m), d_{j}$ is the diameter of link $j(m)$ and $K$ and $\beta$ are obtained by regression analysis. Values of $K(=1020)$ and $\beta(=2)$ for polyethylene pipe available in Australia [15], are used here which give the cost $C_{j}$ in $\$$. For this example, additional installation costs are ignored.

Since pipe diameter is related to flow rate by the expression $d_{j}=\left(4 q_{j} / \pi v\right)^{1 / 2}$, where $v(\mathrm{~m} / \mathrm{s})$ is assumed to be the common velocity of flow in all links and $q_{j}$ is in $\mathrm{m}^{3} / \mathrm{s}$, (1) may be expressed as

$$
C_{j}=\alpha K L_{j} q_{j}^{\beta / 2}
$$


where $\alpha=(4 / \pi v)^{\beta / 2}$.

The sum of the pipe costs for the ring main may now be determined from (2) for each of the eight possible branched networks, or spanning trees. The minimum spanning tree is found for $x=501 / \mathrm{s}\left(q_{4}=0\right)$ with a total cost of $\$ 67634$ if $v$ is taken as $1.8 \mathrm{~m} / \mathrm{s}$.

3.2. Second stage. The principle factor governing pipe flow is the head loss due to friction. The Australian Standard for pipe design, AS 2200 [17] favours the Colebrook-White formula for determining the friction head loss $\left(h_{f}\right)_{j}$ in a link but since this is not expressed in explicit form, it is more convenient to use the empirical Hazen-Williams formula for turbulent flow in the transition zone which is usually expressed in the form

$$
v_{j}=0.354 C_{H} d_{j}^{0.63} S_{j}^{0.54},
$$

where $C_{H}$ is the Hazen-Williams coefficient and $S_{j}$ is the friction head loss per unit length of pipe.

Substituting for $d_{j}$ as before and assuming constant velocity of flow we obtain

$$
\left(h_{f}\right)_{j}=K_{1} \alpha^{-2.435} L_{j} q_{j}^{-0.585},
$$

where $K_{1}=\left(10.68 / C_{H}^{1.85}\right)$ and, for polyethylene pipe, $155 \leq C_{H} \leq 160$ [17].

The loop energy balance law (which is independent of node elevations), may be expressed as $\sum\left(h_{f}\right)_{j}=0$, where each summation is understood to extend over all the links in a loop. Since we make the assumptions for stage two that all links are of the same material and that the link flow velocity is constant, the loop energy balance equation may be expressed in a more simplified form as

$$
F_{\lambda}=\sum L_{j} q_{j}^{-0.585}=0,
$$

where the subscript $\lambda$ identifies the loop. $\ln$ this example there is only one loop, thus $\lambda=1$.

Normally, (5) would be solved by numerical means: however, in this instance, a graphical method is not only possible but more instructive since it enables all the solutions to be inspected. Figure 2 shows $F_{\lambda}$ plotted against $x$, together with the cost function for $v=1.8 \mathrm{~m} / \mathrm{s}$ (which is piece-wise linear because $\beta=2$ ). The eight singularities corresponding to the spanning trees are apparent as are the seven hydraulically feasible solutions located between the singularities. It is clear that the true global optimum corresponds to the minimum spanning tree $(x=50 \mathrm{l} / \mathrm{s})$, but there are two possible hydraulically feasible solutions $(x=39.7 \mathrm{l} / \mathrm{s}$ and $x=59$ $\mathrm{l} / \mathrm{s}$ ) in the neighbourhood of the minimum spanning tree which need to be examined more closely in order to determine the most favourable non-global optimum. Figure 3 


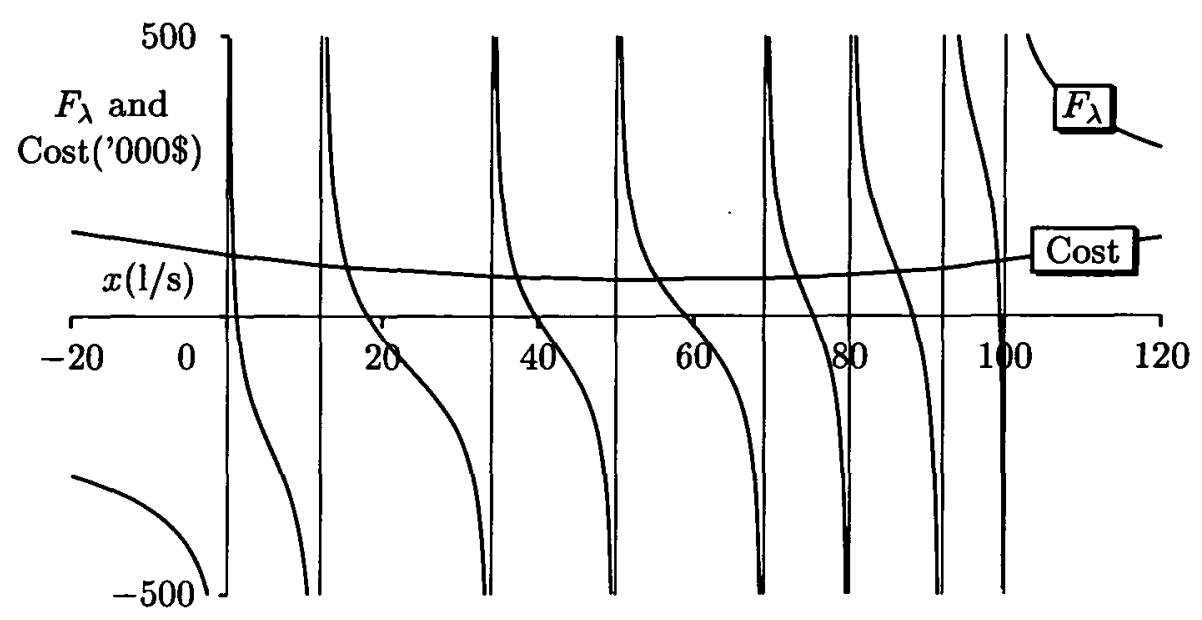

FIGURE 2. Solution of (5) for the ring main.

therefore shows an expanded view of Figure 2 and confirms that the least-cost solution. does not accord with a minimum of the cost function (indeed, the cost function does not have a mathematical minimum), but is found for $x=59 \mathrm{l} / \mathrm{s}$ at a total pipe cost of $\$ 68520$ when $v=1.8 \mathrm{~m} / \mathrm{s}$. The common flow velocity of $1.8 \mathrm{~m} / \mathrm{s}$ originates from the requirement of a minimum residual head of $12 \mathrm{~m}$ at node 5 .

3.3. Third stage. The second stage of the solution results in a set of ideal, or continuous, link diameters. The final step is to replace these diameters with commercial pipe sizes which are available only in discrete intervals. At the same time, the pipe flows must satisfy (5) and the minimum residual head must not be less than $12 \mathrm{~m}$ (it does not have to be exactly $12 \mathrm{~m}$ ). Actual flow velocities are determined from the commercial diameters and the adjusted pipe flows. This stage of the calculation is most conveniently performed on a spreadsheet: details of the procedure have been discussed elsewhere [15].

Although the ring-main example might be considered trivial, it does raise some important matters of principle. The following particular observations are made from this exercise which will be shown to be generally valid in the theoretical discussion which appears next:

(a) the number of spanning trees associated with one loop is equal to the number of links forming the loop;

(b) the number of singularities in the solution space is equal to the number of spanning trees;

(c) the number of hydraulically feasible solutions associated with a loop is one less than the number of links forming the loop; 


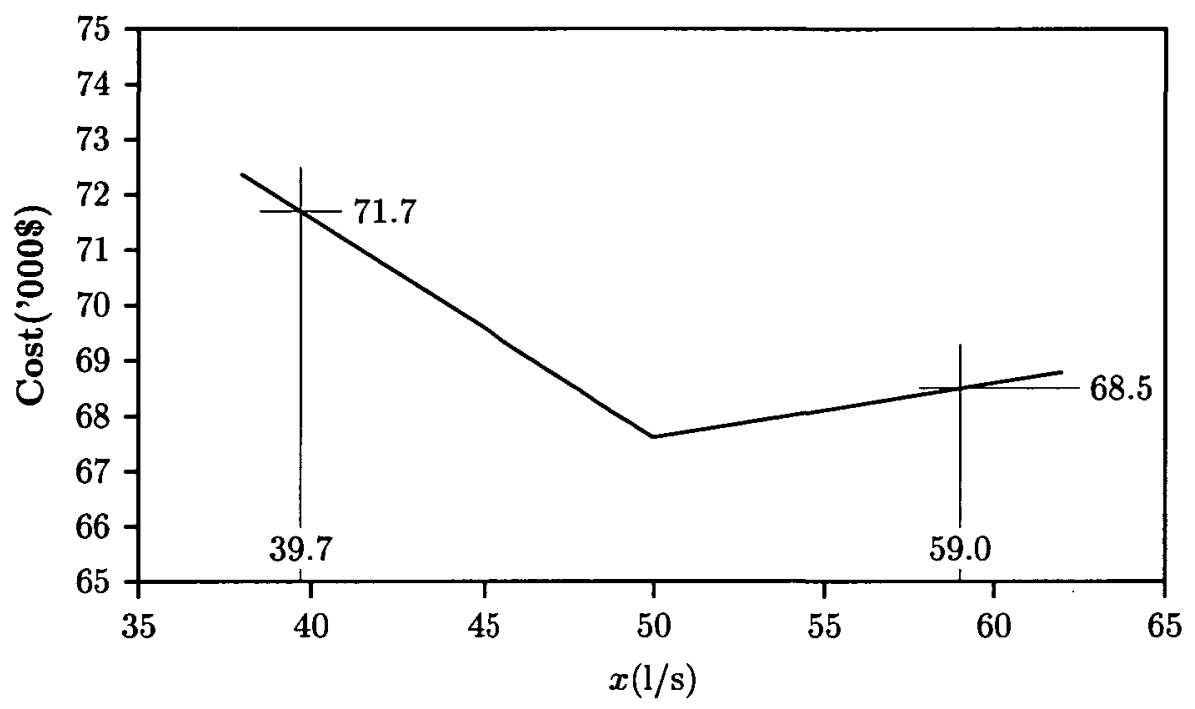

FIGURE 3. Expanded view of the cost function of Figure 2 in the vicinity of the true global optimum.

(d) hydraulically feasible solutions are located in regions of the solution space bounded by singularities;

(e) the dimensionality of the solution space is equal to the number of loops in the network;

(f) neither the true global optimum solution nor the most favourable non-global optimum are associated with a minimum of the cost function;

(g) a mathematical minimum may not exist within the solution space for typical cost functions representing commercial pipes and

(h) the cost function is not continuously differentiable throughout the solution space. The last three observations cast doubts on the validity of the non-linear programming approach to optimisation.

\section{Theory}

In general, a looped water distribution network has $N_{\lambda}$ loops made up of $N_{j}$ links connected by $N_{n}$ nodes such that $N_{j}=N_{n}+N_{\lambda}-1, N_{j} \geq N_{n}>N_{\lambda}$. The nodes are junctions of links which may receive an external supply or deliver a demand. The node supply or demand vector is $\mathbf{Q}_{0}=\left\{Q_{1}, Q_{2}, \ldots, Q_{i}, \ldots, Q_{n}\right\}^{\mathrm{T}}$. The sign convention adopted here assumes supplies to be positive and demands negative; it is also possible for particular $Q_{i}$ to be zero as a special case. The algebraic sum of the $Q_{i}$ for the 
entire network must he zero. The flows in the links $\left(\mathbf{q}=\left\{q_{1}, q_{2}, \ldots, q_{i}, \ldots, q_{j}\right\}^{\mathrm{T}}\right)$ are considered positive if they flow around an arbitrarily chosen loop in a clockwise direction; then, proceeding loop by loop throughout the network, all unassigned flows are similarly designated.

Kirchoff's Laws govern the flows in a looped network. The continuity law requires that the algebraic sum of all the flows into, and out of, a particular node shall be zero and the energy law is satisfied if the algebraic sum of the link friction head-losses around each loop is also zero.

4.1. The continuity equation. A looped network may be considered as a directed linear graph for which the node incidence matrix is represented by $\mathbf{M}_{n}$. The continuity equations for all $N_{n}$ nodes are therefore given by

$$
\mathbf{M}_{n} \cdot \mathbf{q}+\mathbf{Q}_{0}=\mathbf{0},
$$

where $\mathbf{M}_{n}$ is of order $\left\{N_{n} \times N_{j}\right\}, \mathbf{q}$ is of order $\left\{N_{j} \times 1\right\}$ and $\mathbf{Q}_{0}$ is of order $\left\{N_{n} \times 1\right\}$.

Since satisfaction of continuity for the system as a whole is assumed a priori, one of the $N_{n}$ equations in (6) above is redundant and may be chosen arbitrarily for exclusion. The necessary and sufficient conditions for satisfaction of the continuity law for the network may therefore be expressed as

$$
\mathbf{M}_{n-1} \cdot \mathbf{q}+\mathbf{Q}=\mathbf{0},
$$

where $\mathbf{M}_{n-1}$ is the reduced node incidence matrix (of order $\left\{\left(N_{n}-1\right) \times N_{j}\right\}$ ) and $\mathbf{Q}$ (of order $\left.\left\{\left(N_{n}-1\right) \times 1\right\}\right)$ is the corresponding reduced supply/demand vector.

The modified node incidence matrix, $\mathbf{A}=\left|\mathbf{A}_{1} \mathbf{A}_{2}\right|$, is obtained from $\mathbf{M}_{n-1}$ by appropriate interchange of columns such that $\mathbf{A}_{2}$ is square and non-singular. If $\mathbf{q}=\left\{\mathbf{x}, \mathbf{q}_{2}\right\}^{\mathrm{T}}$, (7) may be written in partitioned form as

$$
\left|\mathbf{A}_{1} \mathbf{A}_{2}\right| \cdot\left\{\mathbf{x}, \mathbf{q}_{2}\right\}^{\mathrm{T}}+\mathbf{Q}=\mathbf{0}
$$

thus

$$
\mathbf{q}_{2}=-\mathbf{A}_{2}^{-1} \cdot\left[\mathbf{A}_{1} \mathbf{x}+\mathbf{Q}\right],
$$

where $\mathbf{x}$ is a set of $N_{\lambda}$ independent link flows, $\mathbf{A}_{1}$ is of order $\left\{\left(N_{n}-1\right) \times N_{\lambda}\right\}$ and $\mathbf{A}_{2}$ is of order $\left\{\left(N_{n}-1\right) \times\left(N_{n}-1\right)\right\}$.

Each singularity in the solution space represents zero flow in a particular link and the coordinates of intersections of singularities denote the independent flows $\left(\mathbf{x}_{t}\right)$ in spanning trees. It follows from (9) that if the singularities are identified as $\mathbf{q}=\left\{\mathbf{x}_{t}, \mathbf{q}_{2}\right\}^{\mathrm{T}}=\mathbf{0}$, we have the $N_{j}$ equations in $N_{\lambda}$ unknowns given by

$$
\Delta \cdot \mathbf{x}_{t}=\{\mathbf{0}, \mathbf{P}\}^{\mathrm{T}},
$$


where $\Delta=\{\mathbf{I}, \mathbf{K}\}^{\mathrm{T}}, \mathbf{I}$ is an identity matrix, $\mathbf{K}=-\mathbf{A}_{2}^{-1} \cdot \mathbf{A}_{1}$ and $\mathbf{P}=\mathbf{A}_{2}^{-1} \cdot \mathbf{Q}$.

The overdetermined set of equations (10) are solved for $\mathbf{x}_{t}$ by appropriate methods (discarding inconsistent equations as they arise). The $\mathbf{x}_{t}$ are then substituted for $\mathbf{x}$ in (9) to determine the link flows in all the spanning trees. The cost of each spanning tree is evaluated by reference to a cost function such as that given by (2) and the minimum spanning tree identified.

4.2. The energy equation. The energy equation for the network is

$$
\mathbf{M}_{\lambda} \cdot \mathbf{H}_{f}=\mathbf{0} \text {, }
$$

where $M_{\lambda}$ is the loop incidence matrix for the network and $\mathbf{H}_{f}$ is the link friction head-loss vector obtained from (4). For pipes of the same material and for equal flow velocity in all links, (11) may be simplified to

$$
\mathbf{F}=\mathbf{B} \cdot \mathbf{G}=\mathbf{0},
$$

where $\mathbf{B}$ is the modified loop incidence matrix with columns corresponding to those of $\mathbf{A}, \mathbf{G}=\left\{g_{1}, g_{2}, \ldots, g_{k}, \ldots, g_{j}\right\}^{\mathrm{T}}$ and $g_{k}=L_{k} q_{k}^{-0.585}$.

Equation (12) represents a set of $N_{\lambda}$ non-linear simultaneous equations in $N_{\lambda}$ unknown link flows $\mathbf{x}$. The $\mathbf{x}$ may be determined using a Newton-Raphson recurrence relation given by

$$
\mathbf{x}_{(r+1)}=\mathbf{x}_{(r)}-\mathbf{J}_{(r)}^{-1} \cdot \mathbf{F}_{(r)},
$$

in which $\mathbf{J}(=\mathbf{H} \cdot \Delta)$ is the Jacobian matrix of $\mathbf{F}$ with respect to $\mathbf{x}$.

A typical element of $\mathbf{H}$ is $b_{i k} e_{k}\left(i=1\right.$ to $N_{\lambda}$ and $k=1$ to $N_{j}$ ), where $b_{i k}$ is an element of $\mathbf{B}$ and $e_{k}=-0.585 L_{k} q_{k}^{-1.585}$. The matrix of differential coefficients $\Delta$, $\left(\delta_{k i}=\partial q_{k} / \partial x_{i}\right)$, has already been defined.

The Newton-Raphson iteration scheme requires starting values for $\mathbf{x}$ and these are selected in the neighbourhood of spanning trees identified by the intersection (coordinates $\mathbf{x}_{\boldsymbol{t}}$ ) of the singularities in the solution space. The singularities are edges and their intersections are vertices of a number, $R\left\langle N_{\lambda}\right\rangle$, of closed polygonal convex regions, each of which contains one solution to the set of non-linear equations represented by (12). The most favourable non-global optimum solution is found in the vicinity of the minimum spanning tree.

Spanning trees are obtained by removing one of the links associated with each loop of the original network with the proviso that links common to adjacent loops may be eliminated only once. The number of spanning trees, $T\left\langle N_{\lambda}\right\rangle$ (or the number of ways $N_{\lambda}$ links may be removed to leave a branched network which serves all the nodes of the original looped system), may be determined by consideration of the non-zero elements of the loop incidence matrix, $\mathbf{M}_{\lambda}$, taken row by row. Thus, for example, if 
a network has loops "in-line" and is made up of a constant number of links $r_{\lambda}$, we have a recurrence relation which provides an upper bound for networks having other configurations:

$$
T\left\langle N_{\lambda}\right\rangle=r_{\lambda} T\left\langle N_{\lambda}-1\right\rangle-T\left\langle N_{\lambda}-2\right\rangle,
$$

in which $T(-1\rangle=0, T\langle 0\rangle=1$ and $T\langle 1\rangle=r_{\lambda}$.

All hydraulically feasible solutions have been obtained for rectangular looped networks $\left(r_{\lambda}=4\right)$, with $N_{\lambda}=1,[T\langle 1\rangle=4, R\langle 1\rangle=3] ; N_{\lambda}=2,[T\langle 2\rangle=15, R\langle 2\rangle=$ 9]; $N_{\lambda}=3,[T\langle 3\rangle=56, R\langle 3\rangle=27]$ and for the ring main $\left(r_{\lambda}=8\right)$ with $N_{\lambda}=1$, $[T\langle 1\rangle=8, R\langle 1\rangle=7]$. From these results for $R\left\langle N_{\lambda}\right\rangle$ and the observations at the end of Section 3 , it is possible to deduce a simple relationship for any network with loops "in-line" which is also an upper bound for networks with other configurations:

$$
R\left\langle N_{\lambda}\right\rangle=\prod\left(r_{\lambda}-1\right)_{i} \quad i=1 \text { to } N_{\lambda},
$$

where $r_{\lambda}$ is the number of links associated with a particular loop.

The determination of least-cost solutions for any network is theoretically possible using this approach but it is likely to be time-consuming as $N_{\lambda}$ increases. With loops "in-line" and $r_{\lambda}=4$, for example, $T\langle 4\rangle=209, R\langle 4\rangle=81 ; T\langle 9\rangle=151316, R\langle 9\rangle=$ 19683 and $T\langle 16\rangle=1525870529, R\langle 16\rangle=43046721$. The network layout has an effect on both $T\left\langle N_{\lambda}\right\rangle$ and $R\left\langle N_{\lambda}\right\rangle$, determined by the number of common links between loops. Thus, with $r_{\lambda}=4$ and $N_{\lambda}=4$ (the smallest number of loops for which more than one configuration exists), it is found that $T\langle 4\rangle=192, R\langle 4\rangle=79$ when the loops are arranged in checker-board fashion. This result is explained by the fact that the four-loop "in-line" network loses one link (and hence two possible solutions) when folded back on itself to form a "square" network.

\section{Application of the theory}

Application of the foregoing theory will be illustrated with reference to an example given by Watanatada [13]. This is the analysis and least-cost design of the two-loop triangular network shown in Figure 4. Pipe lengths, supply and demand flows and node elevations are given in the diagram. The supply pressure head at node 4 (initially unspecified) is to be provided by a pump. The choice of commercial pipe sizes and pump must be such that a minimum residual head criterion of $24 \mathrm{~m}$ is satisfied at all nodes. The capital cost of a pump and the recurrent costs of pumping are to be taken into account.

The reduced node incidence matrix for the network is obtained by inspection as

$$
\mathbf{M}_{n-1}=\left|\begin{array}{ccccc}
1 & 0 & -1 & -1 & 0 \\
0 & -1 & 1 & 0 & 1 \\
0 & 0 & 0 & 1 & -1
\end{array}\right| ;
$$




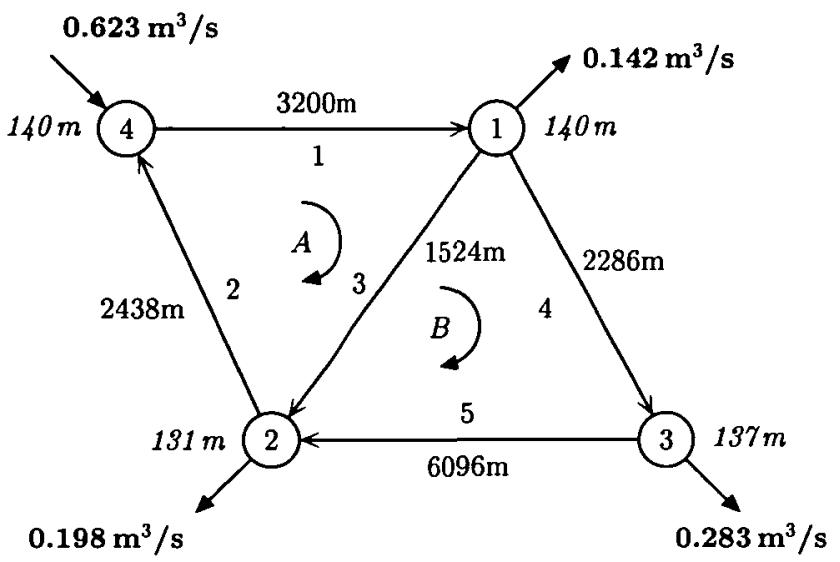

FIGURE 4. Layout of Watanatada's example network.

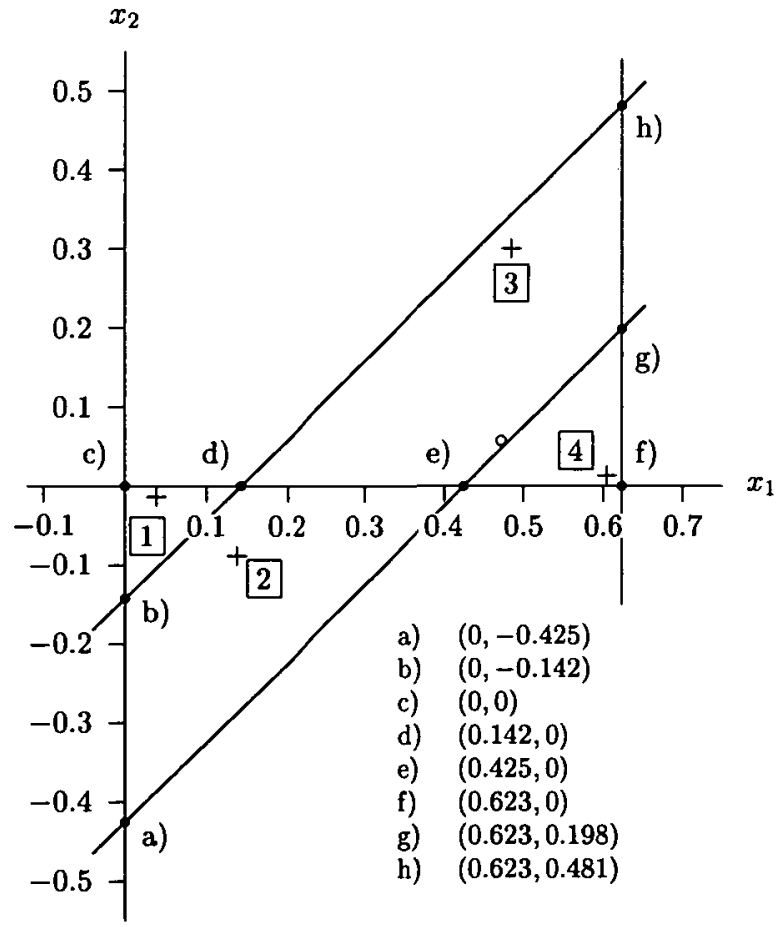

FIGURE 5. Solution space for Watanatada's example network. 
hence,

$$
\mathbf{A}_{1}=\left|\begin{array}{cc}
1 & -1 \\
0 & 1 \\
0 & 0
\end{array}\right| \quad \text { and } \quad \mathbf{A}_{2}=\left|\begin{array}{ccc}
0 & -1 & 0 \\
-1 & 0 & 1 \\
0 & 1 & -1
\end{array}\right|
$$

if the independent flows are $x_{1}=q_{1}$ and $x_{2}=q_{3}$.

Equation (10) now becomes

$$
\left|\begin{array}{cc}
1 & 0 \\
0 & 1 \\
1 & 0 \\
1 & -1 \\
1 & -1
\end{array}\right| *\left|\begin{array}{l}
x_{1} \\
x_{2}
\end{array}\right|=\left|\begin{array}{c}
0 \\
0 \\
0.623 \\
0.142 \\
0.425
\end{array}\right|
$$

since $\mathbf{Q}=\{-0.142,-0.198,-0.283,0.623\}^{\mathrm{T}} \mathrm{m}^{3} / \mathrm{s}$.

The five equations in (16) are the singularities for the network and are shown on the two-dimensional solution space in Figure 5. From (14) with $N_{\lambda}=2$ and $r_{\lambda}=3$ we obtain $T\langle 2\rangle=8$. The eight intersections a) to $h$ ) (coordinates of the spanning trees) are evident from the diagram, but they could equally well have been obtained by solution of (16). From (15) we have $R\langle 2\rangle=4$ and these four closed convex regions (see Figure 5) contain the hydraulically feasible solutions of (12).

The loop incidence matrix may also be obtained by inspection as

$$
\mathbf{M}_{\lambda}=\left|\begin{array}{ccccc}
1 & 1 & 1 & 0 & 0 \\
0 & 0 & -1 & 1 & 1
\end{array}\right|
$$

thus

$$
\mathbf{B}=\left|\begin{array}{ccccc}
1 & 1 & 1 & 0 & 0 \\
0 & -1 & 0 & 1 & 1
\end{array}\right|
$$

The eight link pairs (known as cotrees) which are removed from the network to form spanning trees are obtained from $\mathbf{M}_{\lambda}$, by combining links 1 or 2 (from the first row) together with links 3, 4 or 5 (from the second row) and link 3 (from the first row) with links 4 or 5 (from the second row).

The pipe cost function used by Watanatada was

$$
C_{j}=L_{j}\left(5.01+26.1 d_{j}+52.4 d_{j}^{2}\right),
$$

where $C_{j}$ is in $\$$ if $d_{j}$ and $L_{j}$ are in $\mathrm{m}$.

After evaluating the cost of each spanning tree using (17), it is found that the true global optimum (the minimum spanning tree) for the network is intersection e) in Figure 5 at $\$ 330090$. 
TABLE 1. All solutions from Stage 2 for Watanatada's example ( $C_{H}$ as specified).

\begin{tabular}{|c|c|c|c|c|c|c|c|c|}
\hline Region & $\begin{array}{c}x_{1} \\
\left(\mathrm{~m}^{3} / \mathrm{s}\right)\end{array}$ & $\begin{array}{c}q_{2} \\
\left(\mathrm{~m}^{3} / \mathrm{s}\right)\end{array}$ & $\begin{array}{c}x_{2} \\
\left(\mathrm{~m}^{3} / \mathrm{s}\right)\end{array}$ & $\begin{array}{c}q_{4} \\
\left(\mathrm{~m}^{3} / \mathrm{s}\right)\end{array}$ & $\begin{array}{c}q_{5} \\
\left(\mathrm{~m}^{3} / \mathrm{s}\right)\end{array}$ & $\begin{array}{c}v \\
\left(\mathrm{~m}^{3} / \mathrm{s}\right)\end{array}$ & $\begin{array}{c}\text { Pipe } \\
\text { Cost } \\
(\cdot 000 \$)\end{array}$ & $\begin{array}{c}\text { Total } \\
\text { Cost } \\
\left({ }^{\prime} 000 \$\right)\end{array}$ \\
\hline 2 & 0.147 & -0.476 & -0.099 & 0.103 & -0.180 & 0.90 & 517.1 & 945.8 \\
\hline 4 & 0.606 & -0.01 .7 & 0.014 & 0.450 & 0.167 & 0.99 & 560.2 & 988.9 \\
\hline 3 & 0.505 & -0.118 & 0.323 & 0.040 & -0.243 & 0.86 & 619.7 & 1048.4 \\
\hline 1 & 0.034 & -0.589 & -0.014 & -0.094 & -0.377 & 0.72 & 702.1 & 1130.8 \\
\hline
\end{tabular}

Table 1 shows all four solutions for the network obtained by solving (12) with the recurrence relation from (13). The iteration was terminated when the difference between successive values of the moduli of the vector $\mathbf{x}$ were less than $10^{4}$. The different values of $C_{H}$, for the pipes specified by Watanatada, have been incorporated (as an exception) in the stage two calculation. The solutions are indicated for each region of Figure 5 by plus (+) signs. The common velocities for each solution are dictated by the requirement that the residual head at node 3 should not be less than 24 $\mathrm{m}$. The most favourable solution in terms of pipe cost alone is obtained in Region 2 (see Table 1) at $\$ 517135$. The last column of Table 1 includes the cost of pumps and pumping.

The final optimal design with commercial sizes gives total pipe costs as $\$ 510133$ with flow velocities in the range 0.77 to $1.04 \mathrm{~m} / \mathrm{s}$. The minimum residual head requirement of $24 \mathrm{~m}$ is satisfied at node 3 if the total pressure head at the supply node (node 4) is $171.1 \mathrm{~m}$. The result of Watanatada's calculations for a least pipe diameter of $0.305 \mathrm{~m}$ (12 in) is shown as an open circle close to the minimum spanning tree at e) in Figure 5. Although the total pipe cost is less than that found by the present procedure, Watanatada's design cannot be regarded as realistic since the velocity in link 5 is well below the proscribed minimum.

Watanatada used the following functions to express the cost of a pump and associated equipment, and the recurrent costs of pumping (in \$):

$$
C_{\text {pump }}=2420 Q_{4}^{0.57}\left(H_{4}-z_{4}\right)^{0.87}
$$

and

$$
C_{\text {pumping }}=20300 Q_{4}\left(H_{4}-z_{4}\right) \text {, }
$$

where $Q_{4}$ is the supply at node 4 in $\mathrm{m}^{3} / \mathrm{s}, H_{4}(\mathrm{~m})$ is the total head at node 4 and $z_{4}(\mathrm{~m})$ is the elevation of node 4.

It is now possible to look for a true minimum in the total cost of the system (cost of pipes, pump and pumping). Figure 6 shows how these costs vary with $H_{4}$. The 


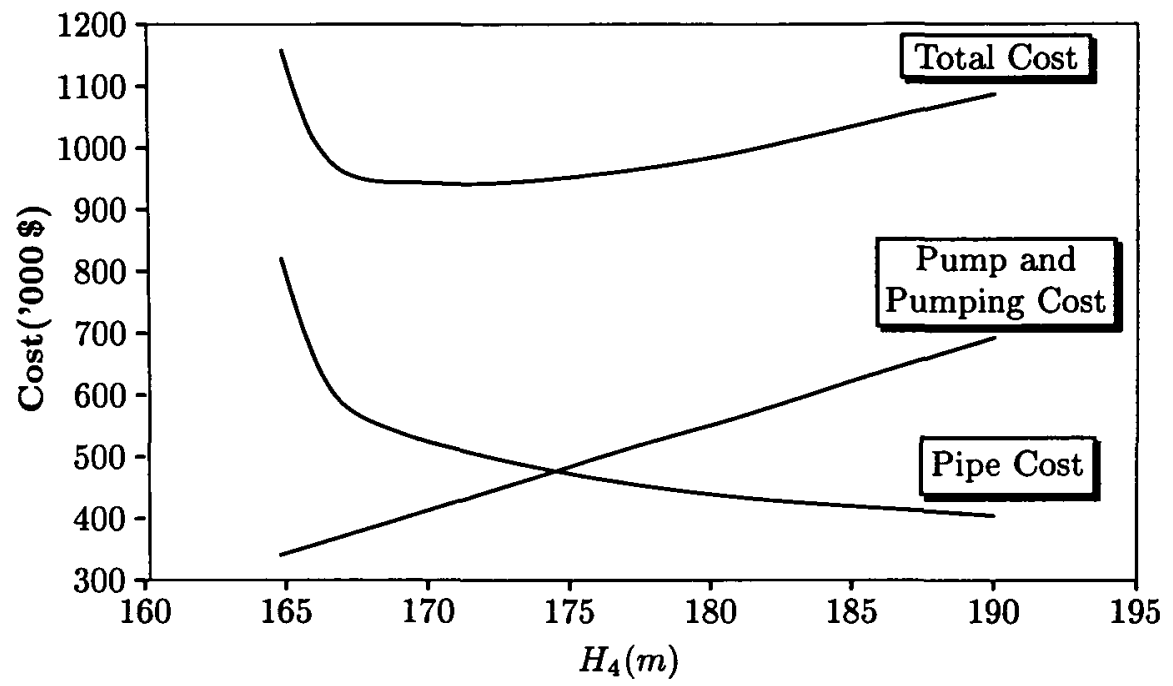

FIGURE 6. Variation of costs with the total head at the supply node for Watanatada's example network.

combined design costs are a minimum at $\$ 939918$ for $H_{4}=171.1 \mathrm{~m}$. Watanatada found a minimum of $\$ 849405$ for $H_{4}=167.8 \mathrm{~m}$ with $v_{5}=0.08 \mathrm{~m} / \mathrm{s}$ when the diameter was constrained to be at least $0.305 \mathrm{~m}$.

\section{Treatment of large networks}

The optimisation procedure is not directly applicable to networks with $N_{\lambda}$ greater than about 5 or 6 . This is partly because the number of spanning trees which have to be investigated, in order to identify the minimum, quickly becomes unmanageable as $N_{\lambda}$ increases; and partly because inversion of the sparse Jacobian found for larger networks consumes excessive amounts of computing resources, even if special techniques are employed. The author has therefore developed a heuristic approach for dealing with large systems in which the network is dismantled into small components which may be conveniently handled. After favourable optimum flows for the separate systems have been found, the components are reassembled to provide a least-cost distribution of flows for the original network.

The deconstruction process [16] has been successfully applied to the network described by Gupta et al. [5] of the National Environmental Engineering Research Institute (NEERI) in India. This medium sized system is the subject of both expansion and upgrading. The original network was served by a single node with an elevated service reservoir and consisted of 10 loops. The upgraded system has 13 loops, three 
additional nodes and six additional links with a single node supplying $96.4 \mathrm{l} / \mathrm{s}$ at peak loading (load factor of 2.5). A second design option is to share the supply between two nodes.

The NEERI network has been divided into three component systems [16]: A (with two rectangular loops and one triangular loop), B (with two rectangular loops) and C (with effectively one rectangular and two triangular loops for the first design option). Link flows in all the spanning trees were first obtained for each component, (A, B and $C$ have respectively 41,15 and 30 spanning trees). These flows were used as starting values for calculating all hydraulically feasible solutions for each sub-system (18 for system A, 9 for B and 12 for C) which were then costed and favourable optima identified. On reassembly, these results enabled stock sizes for new pipes to be selected as necessary (cast iron pipe was used for pipe diameters of $80 \mathrm{~mm}$ and above and galvanised iron for diameters below $80 \mathrm{~mm}$; the minimum permissible pipe size being $50 \mathrm{~mm}$ ). After once again satisfying continuity and energy requirements, new parallel pipes were found to be required for three of the original links in order to attain reasonable values for flow velocities. The total cost of new pipework was US $\$ 24628.65$ and an additional $22.3 \mathrm{~kW}$ booster pump was necessary to cater for peak demand. The non-linear programming approach used by Gupta et al. predicted 22 new parallel pipes costing US $\$ 66228.47$. The design presented here, although requiring a new booster pump as well as new pipework, costs a total of US $\$ 37456.95$ and represents a saving of about 43 percent compared with the NEERI scheme.

For the second design option (supply shared between two nodes), three new parallel pipes were also required. The total cost of new pipework was US\$12 173.89 and two additional booster pumps $(7.8 \mathrm{~kW}$ and $31 \mathrm{~kW})$ were needed to provide for peak demand. Gupta et al. recommended one new pump, 12 new parallel pipes and an elevated service reservoir costing a total of US $\$ 72020.26$. The overall cost of the design obtained by the present method was US $\$ 34422.14$; a saving of about 52 percent compared with the NEERI proposal.

\section{Summary and conclusions}

A procedure for determining least-cost solutions for looped water distribution systems has been described. The method does not involve non-linear programming, but depends on the determination of flows in a minimum spanning tree (the true global optimum) to furnish starting values for the numerical solution of a set of non-linear loop head-loss equations. Although there are a number of hydraulically feasible solutions for a looped network, it is not necessary to find them all since the flow pattern corresponding to the most favourable optimum has been shown to lie in the vicinity of the vertex of closed polygonal convex regions in the solution space whose coordinates are given by independent flows in the minimum spanning 
tree. The boundaries of these regions are singularities identified with zero flows in particular links. The existence of singularities calls into question the validity of the non-linear programming approach which relies on the solution space being continuously differentiable. A further disadvantage of this method is that a minimum of the cost function is sought: a finding of the present study is that a mathematical minimum for typical cost functions associated with commercial pipes may not exist.

A rigorous procedure for finding all spanning trees and all hydraulically feasible solutions for a looped network has been proposed. Previous researchers have offered simplifed algorithms to find approximate minimum spanning trees but with no guarantee that this would lead to a least-cost design. Upper bounds have been determined for the number of spanning trees, $T\left\langle N_{\lambda}\right\rangle$, and for the number of hydraulically feasible solutions, $R\left\langle N_{\lambda}\right\rangle$, in a looped network. The recurrence relation for $T\left\langle N_{\lambda}\right\rangle$ assumes loops to be "in-line" with a constant number of links in each loop. The formula for $R\left\langle N_{\lambda}\right\rangle$ also assumes loops to be "in-line" but the number of links in each loop may vary. Further work is required to study the effect of network configuration on $T\left\langle N_{\lambda}\right\rangle$ and $R\left\langle N_{\lambda}\right\rangle$.

When a looped network is optimised, there is a natural tendency for the system to degenerate into the true global optimum. To prevent this happening, artificial constraints such as limitations on pipe diameters, pipe flows or node pressures have been applied in the past. In the present study, a constant flow velocity in all links has been assumed initially; this has the effect of simplifying the analysis and leads to a more realistic and balanced design. It has been demonstrated that the minimum diameter constraint is unsatisfactory since it may lead to unacceptable values of flow velocity in particular pipes.

The number of spanning trees in a network which have to be investigated in order to find the true global optimum may prohibit direct use of the method for typical complex systems found in practice. Large networks are therefore dismantled, or deconstructed, into components which may be analysed by the present approach. Comparison with existing designs has shown that this heuristic approach is viable and may offer substantial savings in overall project cost.

\section{References}

[1] P. R. Bhave, "Optimal expansion of water distribution systems", J. Environ. Eng. ASCE 111 (1985) 177-197.

[2] A. V. Chiplunkar, S. L. Mehndiratta and P. Khanna, "Looped water distribution systems for single loading", J. Environ. Eng. ASCE 112 (1986) 264-279.

[3] R. Epp and A. G. Fowler, "Efficient code for steady-state flows in networks", J. Hydraulic Division, Proc. ASCE 96 (1970) 43-56. 
[4] R. E. Featherstone and K. K. El-Jumaily, "Optimal diameter selection for pipe networks", $J$. Hydraulic Eng. ASCE 109 (1983) 221-234.

[5] I. Gupta, J. K. Bassin, A. Gupta and P. Khanna, "Optimisation of water distribution system", Environmental Software 8 (1993) 101-113.

[6] S. L. S. Jacoby, "Design of optimal hydraulic networks", J. Hydraulic Division, Proc. ASCE 94 (1968) 641-661.

[7] H. K. Kesavan and M. Chandrashekar, "Graph-theoretic models for pipe network analysis", $J$. Hydraulic Division, Proc. ASCE 98 (1972) 345-364.

[8] D. R. Morgan and I. C. Goulter, "Least-cost layout and design of looped water distribution systems", in Proc. Int. Symp. on Urban Hydrology, Hydraulic and Sediment Control (1982).

[9] G. E. Quindry, E. D. Brill and J. C. Liebman, "Optimisation of looped water distribution systems", J. Environ. Eng. Division, Proc. ASCE 107 (1982) 665-679.

[10] H. J. Rasmusen, "Simplified optimisation of water supply systems", J. Environ. Eng. Division, Proc. ASCE 102 (1976) 313-327.

[11] U. Shamir, "Optimal design and operation of water distribution systems", Water Resources Research 10 (1974) 27-36.

[12] A. B. Templeman, "Discussion on "Optimisation of looped water distribution systems" by Quindry et al. (see [9])", J. Environ. Eng. Division, Proc. ASCE 108 (1982) 599-602.

[13] T. Watanatada, "Least-cost design of water distribution systems", J. Hydraulic division, Proc. ASCE 99 (1973) 1497-1513.

[14] N. B. Webber, Fluid mechanics for civil engineers (Chapman and Hall, London, 1971).

[15] B. W. Young, "Economic design of water distribution systems", Agricultural Engineering Australia (1997).

[16] B. W. Young, "Heuristic approach to the least-cost design of a looped water distribution network", in Int. Conf. on Energy and the Environment (1997).

[17] - Design charts for water supply and sewerage (AS 2200-1978, Standards Assoc. of Australia, 1978). 